\title{
Can small molecule inhibitors of glutaminyl cyclase be used as a therapeutic for Alzheimer's disease?
}

\author{
Haiqiang $\mathrm{Wu}^{*}, 1$ \\ ${ }^{1}$ Department of Pharmacy, School of Medicine, Shenzhen University, Shenzhen, 518060, China \\ * Author for correspondence: Tel.: + 867558617 2799; Fax: +86 7558667 1901; wuhq@szu.edu.cn
}

\begin{abstract}
Alzheimer's disease (AD) is a multifactorial and socioeconomically burdensome disease. In view of the failures of anti-AD candidates, we should try to rethink what we did before and what we should do next, in part at least. Research shows that the more neurotoxic factor, pyroglutamate-A $\beta$, and the more important inflammatory mediators, pyroglutamate-CCL2, both contribute to the initiation of AD specifically and the generation of $\mathrm{N}$-terminal intramolecular cyclization catalyzed by glutaminyl cyclase quality control, the over-expression of which correlates positively with the severity of AD. Subsequently, lowering pyroglutamate- $A \beta s$ and pyroglutamate-CCL2 levels by quality control inhibition using small molecule inhibitors could be expected as an amazing strategy for the prevention and treatment of AD.
\end{abstract}

First draft submitted: 21 August 2017; Accepted for publication: 31 August 2017; Published online: 27 October 2017

Keywords: Alzheimer's disease $\bullet$ glutaminyl cyclase $\bullet$ inhibitors

Alzheimer's disease $(\mathrm{AD})$ is a systematically chronic neurodegenerative disease characterized by diminution of cognitive function, memory, neurobehavioral manifestations, social withdrawal and other behavioral symptoms. $\mathrm{AD}$ is the most common cause of dementia in aging and accounts for $60-80 \%$ of all cases. The prevalence of $\mathrm{AD}$ has increased dramatically over the past decade and is expected to become even worse in the future owing to increased life expectancy. Unfortunately, we have almost no idea about the exact pathology of this multifactorial disease, and there is no cure, at least no disease modifying agents, for this socioeconomically burdensome disease. For these reasons, demand for development of appropriate prevention and/or treatment options for $\mathrm{AD}$ will continue to grow rapidly.

As one of the hallmarks, extracellular amyloid plaques contribute to the death of neurons and development of $\mathrm{AD}$. $\beta$-Amyloid $(\mathrm{A} \beta)$ pathology is well documented, and the $\mathrm{A} \beta$ cascade hypothesis has been one of the main hypotheses. However, the fact is no candidate targeting $A \beta$ pathology directly has passed through clinical trials including the failure of Lilly's solanezumab and Merck's verubecestat. Obviously, the development of anti-AD agents is a world challenge now. We cannot judge that the amyloid cascade hypothesis is flawed even now, but is $\mathrm{A} \beta$ aggregate the reasonable target for the discovery of anti-AD agents? Do we have any 'misunderstanding' about the $\mathrm{AD}$ pathology? What is the problem with the development strategy we use? Maybe it is the time for us to change our chair and rethink in part at least what we saw, what we did, what we learned and what we should do next.

Many studies confirm that the formation of $A \beta$ plaques, mainly composed of $A \beta_{42}$ and $A \beta_{40}$, has no direct effects on the development of neurodegeneration and other clinical AD symptoms. These plaques can be detected in both $\mathrm{AD}$ brains and normal brains. What is the pathological difference between these two types of brain? In recent years, a variety of $N$-truncated $A \beta$ s have been identified only in $A D$ brains including pyroglutamate- $A \beta s$ (pE-A $\beta s$ ), which are the generations of $A \beta$ s undergoing $\mathrm{N}$-terminal truncation by two or ten amino acids and following cyclization of resulting $\mathrm{N}$-terminal glutamate $(\mathrm{E})$ to pyroglutamate $(\mathrm{pE})$. These $\mathrm{pE}-\mathrm{A} \beta \mathrm{s}$, especially $\mathrm{pE}-\mathrm{A} \beta_{3-40 / 42}$, account for more than $50 \%$ of the total plaques in $\mathrm{AD}$ brains [1]. Compared with $\mathrm{A} \beta \mathrm{s}$, $\mathrm{pE}-\mathrm{A} \beta$ s confer proteolytic resistance, increased hydrophobicity, aggregate more rapidly and seed further $A \beta$ aggregation. Meanwhile, $p E-A \beta$ can drive the downstream toxicity cascade to destroy the plasticity of synapses and induce the death of neurons through the formation of much stronger neurotoxic oligomers and 'infection' of Tau, ROS, $\mathrm{Ca}^{2+}$ and other pathways [2-4]. 
These findings suggest that $\mathrm{pE}-\mathrm{A} \beta$ s may be one of the real causes of $\mathrm{AD}$, especially in the early stage before the $\mathrm{A} \beta$ aggregates could be detected. Inhibiting the generation of $\mathrm{pE}-\mathrm{A} \beta \mathrm{s}$ is a good option for the development of anti-AD agents because of $\mathrm{pE}-\mathrm{A} \beta \mathrm{s}^{\prime}$ crucial role.

Inflammation is another important risk component in the initiation of $\mathrm{AD}$. Although, we do not know the exact mechanism yet [5]. Neuroinflammation is clinically characterized by activated microglia and astrocytes surrounding the plaques in the brain. Among the inflammatory mediators, CCL2, also known as MCP-1 mainly recruits monocytes and macrophages and exhibits its biological activity through the binding with CCR2. CCL2 attributes to the activation of microglia and astrocytes, in turn, activated microglia and astrocytes can secrete more CCL2 and other inflammatory molecules. Decreasing CCL2 level may contribute to treatment of inflammatory diseases, including $\mathrm{AD}[6,7]$. However, $\mathrm{N}$-terminal modified CCL2, pE-CCL2, may be the more important inflammatory component in the course of AD compared with CCL2. This cyclized form of CCL2 is also generated by N-terminal cyclization of resulting $\mathrm{E}$ to $\mathrm{pE}$, which confers resistance against degradation by aminopeptidase and is more active in chemotaxis assays. As a result, pE-CCL2 exhibits much more functional CCL2 activity in vivo [8]. The generation of pE-CCL2 is initiated in AD patients decades before the formation of $A \beta$ fibrils. And the pE-CCL2 levels in cerebrospinal fluid correlates with the faster cognitive decline in prodromal AD patients. Accordingly, inhibiting the generation of pE-CCL2 is believed to be a promising new approach for the prevention and treatment of neuroinflammation at the beginning of $\mathrm{AD}[9]$.

Considering the specific presence of $\mathrm{pE}-\mathrm{A} \beta$ s and $\mathrm{pE}-\mathrm{CCL} 2$ and the key role during the initiation and development of $\mathrm{AD}$, lowering $\mathrm{pE}-\mathrm{A} \beta \mathrm{s}$ and $\mathrm{pE}-\mathrm{CCL} 2$ levels by inhibiting their generation, could be expected as an amazing strategy for the discovery of novel anti-AD chemicals, and method for the prevention and treatment of $\mathrm{AD}$. $\mathrm{pE}-\mathrm{A} \beta \mathrm{s}$ and pE-CCL2 are both the generations of $\mathrm{N}$-terminal intramolecular cyclization. Normally, this cyclization of peptide/protein $\mathrm{N}$-terminal glutamine residues into $\mathrm{pE}$ is important and a common post-translational event for maturation of bioactive neuropeptides, hormones and cytokines in the secretory pathway. This cyclization was thought to be a spontaneous reaction, however, it has now been approved that this conversion is mainly catalyzed by glutaminyl cyclase (QC, also known as QPCT, EC 2.5.2.3), which is widely distributed in mammalian brain with robust expression in the hippocampus and cortex. The higher expression of QC is directly associated with several complex pathologies ranging from inflammation to neurodegenerative diseases. The generation of $\mathrm{pE}-\mathrm{A} \beta \mathrm{s}$ and pE-CCL2 are both catalyzed by the over expressed QC, and QC expression level correlates positively with the accumulation of $\mathrm{pE}-\mathrm{A} \beta s$ and $\mathrm{pE}-\mathrm{CCL} 2$ in $\mathrm{AD}$ brains and the severity of $\mathrm{AD}[8,10]$. Furthermore, compared with age-matched normal brains, much higher QC mRNA levels are found in $\mathrm{AD}$, and the higher expression of QC can even be seen in peripheral blood before any other AD related biomarkers can be detected [11]. According to this research, QC inhibition may offer a new opportunity for the prevention and treatment of $\mathrm{AD}$ by inhibiting the generation of $\mathrm{pE}-\mathrm{A} \beta \mathrm{s}$ and $\mathrm{pE}-\mathrm{CCL} 2$ and blocking the related pathway consequently in the earliest stage.

To inhibit the activity of QC, a few small molecule QC inhibitors have been reported [12-16]. These chemicals contain an aromatic motif tethered to an imidazole moiety, where the aromatic ring matches the hydrophobic space at the entrance of the active site and the imidazole moiety binds the catalytic zinc ion at the bottom of the pocket. These inhibitors were found to be efficient in reducing the generation of pE-A $\beta$ s and pE-CCL2 in vitro and in vivo. Further assessments corroborated that these compounds can reduce monocyte/macrophage infiltration, exhibit strong anti-inflammatory actions in many inflammation models, and improve the behavior and reduce AD pathology in several transgenic mouse models. Meanwhile, it is inspiring to see that PQ912, a competitive inhibitor of QC, inhibits QC activity in cerebrospinal fluid by $92 \%$, exhibits a benefit on working memory and a trend on attention in Phase II SAPHIR trial. The synaptic marker neurogranin and the inflammatory marker YKL40 trend downward after the treatment of PQ912 in these enrolled people with mild cognitive impairment or mild dementia [17]. So, small molecule inhibitors of QC may represent an alternative therapeutic strategy to treat AD.

As we know, blockade of $\mathrm{pE}$ formation by inhibiting QC may have unpredictable side effects because $\mathrm{pE}$ is also the $\mathrm{N}$-terminal of many other proteins, hormones and peptides. With this potential issue in mind, discovery of inhibitors with a novel skeleton and further understanding of QC biology will hopefully lead to the development of disease-modifying agents for the treatment of $\mathrm{AD}$.

Financial \& competing interests disclosure

This work was supported by the grants of National Natural Science Foundation of China (No. 81573288), the Science and Technology Planning Project of Guangdong Province (No. 2013B021100022) and the Science and Technology Planning Project of Shenzhen City (No. JCYJ20170302144938485). The author has no other relevant affiliations or financial involvement with any organization 
or entity with a financial interest in or financial conflict with the subject matter or materials discussed in the manuscript apart from those disclosed.

No writing assistance was utilized in the production of this manuscript.

\section{References}

1. Wirths O, Hillmann A, Pradier L, Hartig W, Bayer TA. Oligomeric pyroglutamate amyloid- $\beta$ is present in microglia and a subfraction of vessels in patients with Alzheimer's disease: implications for immunotherapy. J. Alzheimer's Dis. 35(4), 741-749 (2013).

2. Gunn AP, Masters CL, Cherny RA. Pyroglutamate-Aß: role in the natural history of Alzheimer's disease. Int. J. Biochem. Cell Biol. 42(12), 1915-1918 (2010).

3. Schlenzig D, Manhart S, Cinar Y et al. Pyroglutamate formation influences solubility and amyloidogenicity of amyloid peptides. Biochemistry 48(29), 7072-7078 (2009).

4. Nussbaum JM, Schilling S, Cynis $\mathrm{H}$ et al. Prion-like behaviour and tau-dependent cytotoxicity of pyroglutamylated amyloid- $\beta$. Nature 485(7400), 651-655 (2012).

5. Bagyinszky E, Giau VV, Shim K, Suk K, An SSA, Kim S. Role of inflammatory molecules in the Alzheimer's disease progression and diagnosis. J. Neurol. Sci. 376, 242-254 (2017).

6. Bose S, Cho J. Role of chemokine CCL2 and its receptor CCR2 in neurodegenerative diseases. J. Arch. Pharm. Res. 36(9), 1039-1050 (2013).

7. Kiyota T, Gendelman HE, Weir RA, Higgins EE, Zhang G, Jain M. CCL2 affects $\beta$-amyloidosis and progressive neurocognitive dysfunction in a mouse model of Alzheimer's disease. Neurobiol. Aging 34(4), 1060-1068 (2013).

8. Cynis H, Hoffmann T, Friedrich D et al. The isoenzyme of glutaminyl cyclase is an important regulator of monocyte infiltration under inflammatory conditions. EMBO Mol. Med. 3(9), 545-558 (2011).

9. Chen YL, Huang KF, Kuo WC, Lo YC, Lee YM, Wang AH. Inhibition of glutaminyl cyclase attenuates cell migration modulated by monocyte chemoattractant proteins. Biochem. J. 442(2), 403-412 (2012).

10. Schilling S, Hoffmann T, Manhart S, Hoffmann M, Demuth HU. Glutaminyl cyclases unfold glutamyl cyclase activity under mild acid conditions. FEBS Lett. 563(1-3), 191-196 (2004).

11. Valenti MT, Bolognin S, Zanatta C et al. Increased glutaminyl cyclase expression in peripheral blood of Alzheimer's disease patients. J. Alzheimer's Dis. 34(1), 263-271 (2013).

12. Buchholz M, Heiser U, Schilling S, Niestroj AJ, Zunkel K, Demuth HU. The first potent inhibitors for human glutaminyl cyclase: synthesis and structure-activity relationship. J. Med. Chem. 49(2), 664-677 (2006).

13. Buchholz M, Hamann A, Aust $\mathrm{S}$ et al. Inhibitors for human glutaminyl cyclase by structure based design and bioisosteric replacement. J. Med. Chem. 52(22), 7069-7080 (2009).

14. Ramsbeck D, Buchholz M, Koch B et al. Structure-activity relationships of benzimidazole-based glutaminyl cyclase inhibitors featuring a heteroaryl scaffold. J. Med. Chem. 56(17), 6613-6625 (2013).

15. Tran PT, Hoang VH, Thorat SA et al. Structure-activity relationship of human glutaminyl cyclase inhibitors having an N-(5-methyl-1H-imidazol-1-yl)propyl thiourea template. Bioorg. Med. Chem. 21(13), 3821-3830 (2013).

16. Li M, Dong Y, Yu X et al. Synthesis and evaluation of diphenyl conjugated imidazole derivatives as potential glutaminyl cyclase inhibitors for treatment of Alzheimer's disease. J. Med. Chem. 60(15), 6664-6677 (2017).

17. Alzforum. www.alzforum.org/therapeutics/pq912 
